\title{
Isolation of Markers from Recently Transposed LINE-1 Retrotransposons
}

BioTechniques 29:1310-1316 (December 2000)

\author{
Michael A. Cantrell, Robert \\ A. Grahn, LuAnn Scott and \\ Holly A. Wichman \\ University of Idaho, Moscow, \\ ID, USA
}

\section{INTRODUCTION}

Eukaryotic genomes contain a range of retroelements such as LINE-1s (L1s) and endogenous retroviruses $(2,6,8)$. A common complicating factor in studies of these elements is their large copy number, ranging in L1 elements up to greater than 100000 copies per mammalian genome and composing up to $20 \%$ of these genomes. Random sam pling of retroelements can lead to inaccurate conclusions about their nature and activity because the vast majority may be inactive pseudogenes that inserted long ago and have collected mutations over great periods of time. For example, random sampling of L1 elements in two species of rodents resulted in the erroneous conclusion $(10,20)$, which was later refuted $(4,16)$, that L1 transposition had slowed dramatically or ceased in these species. Previous techniques aimed at analysis of recently inserted L1 elements suffered from problems such as the need for sequencing extensive numbers of elements, the requirement of a chance encounter with a recent phenotypically observable insertion event, or laborious approaches targeting specific subfamilies $(4,5,11,18)$. In this paper, we describe a technique that allows easy isolation of a portion of L1s from a broad range of mammalian species, followed by identification of those elements that have recently inserted. This general method should be applicable to studies of any other retroelements that contain coding regions.

The method is based on two observations. First, regions of the reverse transcriptases of retroelements are highly conserved so that the design of appropriate degenerate primers allows PCR am plification of a wide range of these elements (19). Second, these elements collect random mutations as time passes after insertion, so more recently inserted (younger) elements will have fewer mutations and should be more likely to retain their open reading frames (ORFs).

For this paper, we designed degenerate PCR primers based on conserved amino acid sequences in the $\mathrm{L} 1$ reverse transcriptases from a wide range of mammalian L1s because the reverse transcriptase reading frame is the most highly conserved region in L1 elements. We then modified a standard bacterial cloning vector such that it does not contain a functional $l a c Z^{\prime}$ reading frame but can produce a functional LacZ' fusion peptide if an L1 ORF amplified with the degenerate primers is inserted appropriately. By careful choice of the recipient bacterial strain and modification of lacZ induction conditions, we have produced a blue/white colony screen for delineation of blue colonies containing L1s that have transposed so recently that they still contain ORFs. White colonies can be used as a source of old elements (ones that have transposed long ago).

\section{MATERIALS AND METHODS}

\section{Degenerate Primer Design and PCR Amplification}

A typical full-length L1 element and the region amplified are shown in Figure 1. The upstream primer was designed to include the highly conserved Y/FXDD box from homology domain 5 in a published alignment of reverse transcriptases from viruses and trans- 
posable elements (22). The downstream primer, 9Rdeg, was designed to a region within the L1 ORF 2 but outside of the reverse transcriptase domain. Final design of primers was based on our alignments of L1s from human, gorilla, mouse, rat, deer mouse, slow loris, rabbit, and dog. Figure 2A shows the pertinent regions from an alignment of L1s from six of these species, while Figure $2 \mathrm{~B}$ shows the degenerate primers used for PCR amplification aligned with the amino acid sequence upon which they are based. Even though the alignment in Figure $2 \mathrm{~A}$ shows only Fs at amino acid number 707, the 8FDeg primer was designed for the possibility of Fs or Ys at this position because a large number of reverse transcriptases contain Ys at this position. The $\mathrm{E}$ rather than $\mathrm{a} \mathrm{D}$ in the dog sequence at position 710 was deemed to be due to a mutation after insertion because the two Ds at this location are the most highly conserved amino acid pair in reverse transcriptases and have been found in the vast majority of all functional reverse transcriptases (22). Degeneracy was kept under 200-fold by limited use of inosines. 8FDeg and 9RDeg degeneracies are 32- and 192-fold, respectively. Inosines were placed as far from the $3^{\prime}$ ends of primers as possible because $3^{\prime}$ ends are important for annealing specificity, and although inosine can base pair with any of the four nucleotide bases, it does not exhibit the same binding affinity for each base and can therefore introduce a bias for some sequence subsets $(13,17)$. Two restriction sites were added at the $5^{\prime}$ end of each primer to increase cloning flexibility and to allow higher annealing temperatures after the initial PCR cycles.

Degenerate PCR amplifications of $50 \mu \mathrm{L}$ contained $10 \mathrm{mM}$ Tris- $\mathrm{HCl}, \mathrm{pH}$ 8.3, $50 \mathrm{mM} \mathrm{KCl}, 2 \mathrm{mM} \mathrm{MgCl}_{2}, 200$ $\mu \mathrm{M}$ each dNTP, 100 pmol each primer, $200 \mathrm{ng}$ genomic DNA, and $2.5 \mathrm{U}$ AmpliTaq ${ }^{\circledR}$ DNA polymerase (Applied Biosystems, Foster City, CA, USA). Hot starts were performed by combining all components except the genomic DNA and the polymerase, heating to $70^{\circ} \mathrm{C}$, and then adding these final two components before cycling.

Amplification conditions were designed with a low initial annealing tem perature to maximize the range and age of L1s that would be amplified. This temperature of $41.8^{\circ} \mathrm{C}$ is based on only the portion of each primer that is homologous to $\mathrm{L} 1$ sequences and is $5^{\circ} \mathrm{C}$ below the melting temperature $\left(\mathrm{T}_{\mathrm{m}}\right)$ of that region when it has the maximum $\mathrm{A}+\mathrm{T}$ content within the degenerate mixture $\left(\mathrm{T}_{\mathrm{m}} \mathrm{ATmax}-5^{\circ}\right)(15)$. A slow ramp of $1^{\circ} \mathrm{C} / 5 \mathrm{~s}$ was used for the first five cycles to increase annealing of L1s with higher $\mathrm{T}_{\mathrm{m}} \mathrm{s}$. After the first five cycles, the annealing temperature was raised to the $\mathrm{T}_{\mathrm{m}} \mathrm{ATmax}-7^{\circ}$ for the total length of each primer (including bases beyond the region of L1 homology). Thermal cycling was performed in a GeneAmp ${ }^{\circledR}$ PCR System 9600 (Applied Biosystems) as follows: hot start at $70^{\circ} \mathrm{C}, 1.5 \mathrm{~min}$ denaturation at $94^{\circ} \mathrm{C}, 5$ cycles of $94^{\circ} \mathrm{C}$ for 30 $\mathrm{s}, 41.8^{\circ} \mathrm{C}$ for $30 \mathrm{~s}, 2.5 \mathrm{~min}$ ramp to $72^{\circ} \mathrm{C}$, and $72^{\circ} \mathrm{C}$ for $1 \mathrm{~min}$, followed by 23 cycles of $94^{\circ} \mathrm{C}$ for $30 \mathrm{~s}, 56.6^{\circ} \mathrm{C}$ for $30 \mathrm{~s}$, and $72^{\circ} \mathrm{C}$ for $1 \mathrm{~min}$, followed by a final elongation at $72^{\circ} \mathrm{C}$ for $7 \mathrm{~min}$. In some cases, with either very divergent species or when dealing with only exceptionally old $\mathrm{L} 1 \mathrm{~s}$, the $\mathrm{T}_{\mathrm{m}}$ for the first five cycles was reduced to $37.8^{\circ} \mathrm{C}$, the ramp was kept at $1^{\circ} \mathrm{C} / 5 \mathrm{~s}$, and the number of cycles at the higher temperature was increased from 23 to 28 to increase the amount of product and potentially collect more divergent sequences.

\section{Construction of Cloning Vector and Libraries}

The plasmid pBluescript ${ }^{\circledR}$ II $\mathrm{KS}+$ (Stratagene, La Jolla, CA, USA) was modified to produce the plasmid vector pKSW, which does not contain a functional lac $Z^{\prime}$ ORF, but which can produce a functional LacZ alpha peptide fusion protein if an appropriate fragment is inserted to recreate an ORF. The pBS II KS+ plasmid was digested with EcoRI and NotI. The complementary oligonucleotides, 5'-AATTCACCCG GGGGATCCTTCTAGAGC-3' and 5'GGCCGCTCTAGAAGGATCCCCCG GGTG-3', were then annealed and ligated into the digested plasmid, producing $\mathrm{pKSW}$. This new vector allows the directional insertion of PCR products produced with the 8 FDeg and 9RDeg primers after digestion with either EcoRI or XhoI for the $5^{\prime}$ end of the insert plus $B$ amHI for the $3^{\prime}$ end of the insert. The additional option of a PstI cloning site is available for the $3^{\prime}$ end of the insert when the pKSW vector is prepared from a clone that contains an 8FDeg/9RDeg-generated L1. When an amplification product from an L1 element with an ORF is inserted, a fusion protein is produced from the lacZ initia-

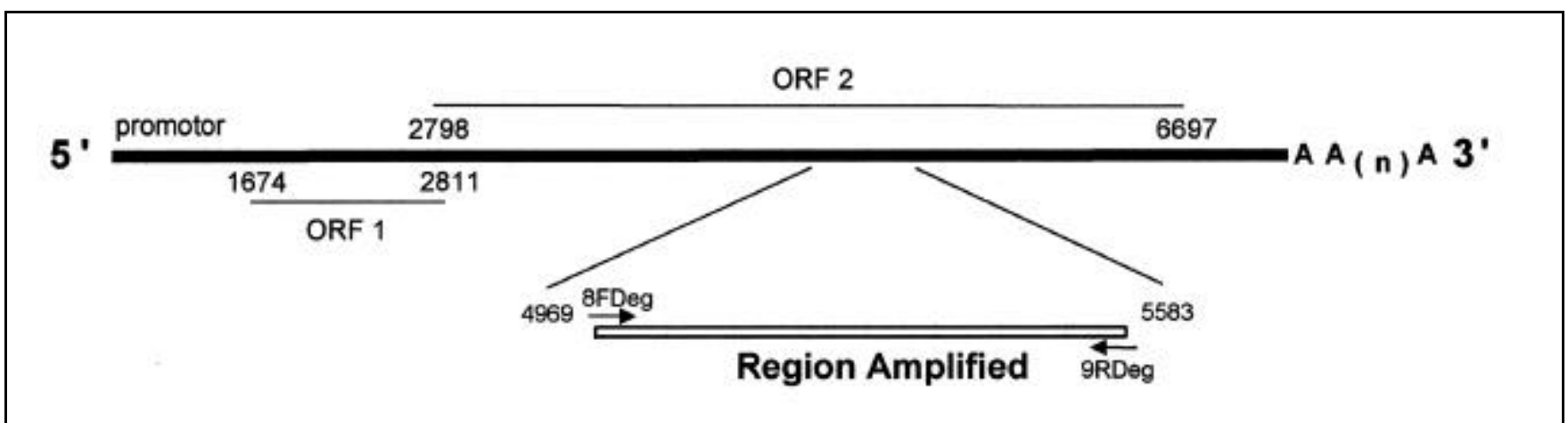

Figure 1. Map of a typical full-length L1 element. Some major features of a typical full-length L1 element with nucleotide numbering based on the mouse element described by Loeb et al. (12), GenBank ${ }^{\circledR}$ accession no. M13002. ORF 2 is the reverse transciptase/endonuclease reading frame. The numbers defining the amplified region include only the regions of homology in the 8FDeg and 9RDeg primers. 


\section{Research Report}

tion codon upstream of the multiple cloning site, containing 205 amino acids of L1 reverse transcriptase sequence fused with the LacZ alpha peptide.

Amplified DNA was purified with the QIAquick ${ }^{\mathrm{TM}}$ PCR Purification Kit (Qiagen, Valencia, CA, USA) before extensive digestion with EcoRI and B anHI. The 630-bp digestion product was then isolated following gel electrophoresis by standard techniques (1). The DNA was ligated into pKSW, also cut with EcoRI and $B$ anHI, and subsequently electroporated into $E$. coli strain TG1 (Stratagene).

\section{Colony Screening Assay}

Plasmids to be screened were electroporated into E. coli strain TG1. Cells were then plated onto LB plates containing $100 \mu \mathrm{g} / \mathrm{mL}$ ampicillin, $40 \mu \mathrm{g} / \mathrm{mL} \mathrm{X-}$ gal, and $0.05 \mathrm{mM}$ isopropyl- $\beta$-D-thiogalactoside (IPTG) (the last two from Life Technologies, Rockville, MD,
USA) and incubated for $16-18 \mathrm{~h}$ at $37^{\circ} \mathrm{C}$ before scoring. It was essential to use plates within one week of pouring (stored at $4^{\circ} \mathrm{C}$ ) and to score only isolated colonies incubated as described above. Scoring was most reproducible when colonies were observed on a background of light blue paper, type PIMP2201-BE (Boise Cascade, Boise, ID, USA). Positive controls used during development of the colony screening assay consisted of a clone containing pBluescript II KS+ and a number of clones containing the pKSW vector with the appropriate regions of previously isolated Peromyscus $\mathrm{ORF}^{+}$L1s inserted. Negative controls were a clone containing pKSW plus a number of clones containing $\mathrm{pKSW}$ constructs that had received the appropriate regions of $\mathrm{ORF}^{-} \mathrm{L} 1 \mathrm{~s}$.

\section{RESULTS AND DISCUSSION}

Before using degenerate primers to amplify part of the reverse transcriptase gene from a broad range of L1s, we inserted that same region from a number of previously isolated L1s into the screening vector, pKSW, such that only those clones containing ORFs should give rise to blue colonies upon screening with X-gal and IPTG. Tests for ORFs (i.e., blue vs. white colonies) in the $E$. coli strain DH5 $\alpha$ revealed an unacceptable percentage of both false-positive and false-negative clones even when the scoring conditions were carefully controlled. Modifications of growth time and X-gal/IPTG concentrations, combined with tests of different bacterial strains, were essential to produce the assay described in Materials and Methods in which greater than $98 \%$ of the colonies from any of the test constructs were of the predicted color. It is extremely important that the conditions described be maintained, particularly when comparisons are made between species.

The degenerate primers (Figure 2)

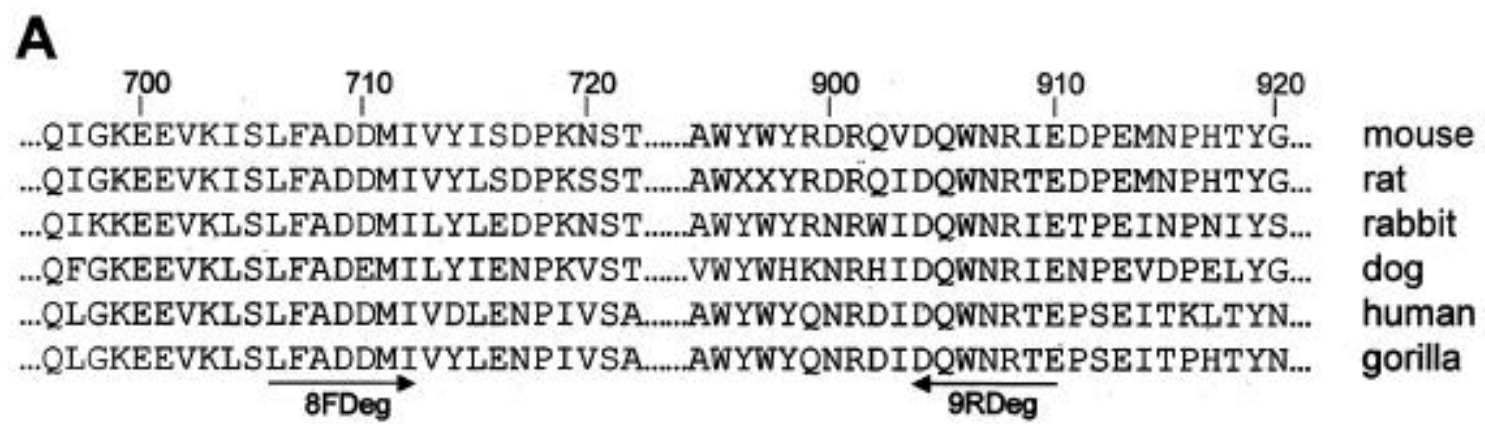

B

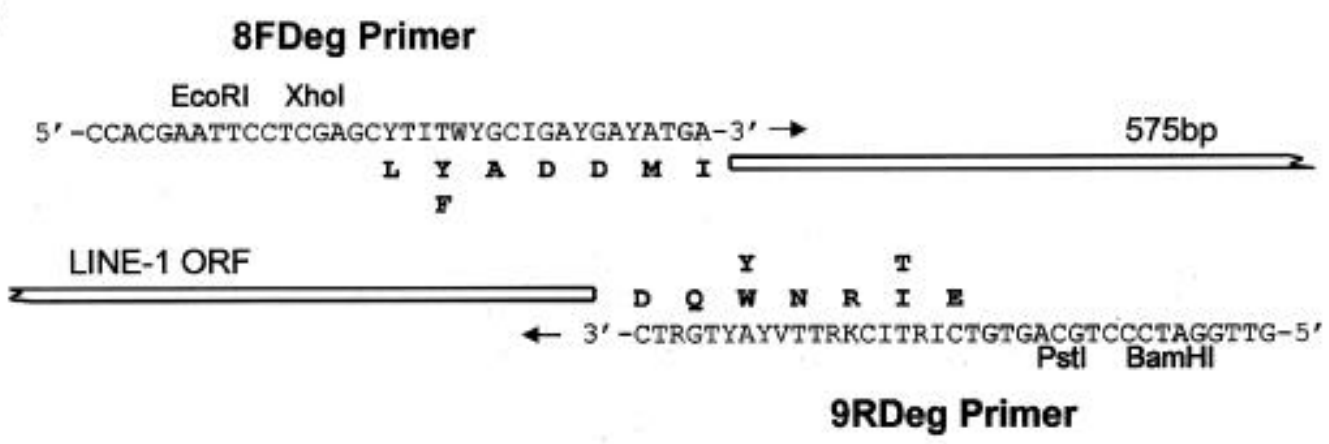

Figure 2. Design of degenerate PCR primers. (A) Alignment of six mammalian L1 reverse transcriptases showing the region used for primer design and 10 amino acids upstream and downstream of each primer. Numbering of amino acids is based on the second ORF of the mouse sequence (14), GenBank accession no. U15647. Accession numbers for other sequences are: rat, M13100; rabbit, X15965; dog, AB012223; human, M19503; and gorilla, AFO36235. Xs in the rat sequence represent a deletion in that sequence. (B) Diagram of the 8FDeg and 9RDeg degenerate primers used to amplify 575 bp of L1 elements. Restriction enzyme sites added near the $5^{\prime}$ end of each primer are shown. The $3^{\prime}$ portion of each primer homologous with L1 reverse transcriptase genes is aligned with the corresponding amino acid sequence (shown in bold). The broken bar indicates the approximately 575-bp ORF between the primers that is amplified from the L1 reverse transcriptase genes. 
were designed, primarily, to allow am plification of L1 sequences from a wide range of rodent species and, secondarily, to allow amplification from a wide range of other mammalian species. They were initially used to amplify genomic DNA from the deer mouse, Peromyscus maniculatus. The major amplification products of approximately $630 \mathrm{bp}$ from two completely independent amplifications were inserted into pKSW, giving rise to two libraries. Each of these libraries showed a similar percentage of blue clones. Thirty-one percent of the clones were blue in one library and $27 \%$ in the other library. A number of blue and white colonies were randomly selected from each of these libraries for further analysis, and $100 \%$ of them were found to contain the predicted L1 region. Eighteen elements from blue clones and 14 from white clones were sequenced. ORFs were found in the majority of the blue clones $(94 \%)$ but only in a minority of the white clones $(21 \%)$. This contrasts greatly with previous studies of L1 elements in a range of mammals. In some studies, only elements with interrupted ORFs have been identified, while in other studies, elements with ORFs have been identified only after sequencing a relatively large number of clones or using laborious approaches tailored for identification of such elements $(4,5,10$, $11,18,20)$. The high incidence of ORFs among clones isolated after this degenerate PCR, even if no color screen had been used, underscores the power of this PCR alone to enrich for recently inserted elements. However, the enrichment due to degenerate PCR alone varies between species. Blue/white screening will be most useful to characterize species in which recent L1 activity is reduced or absent.

The presence of $\mathrm{ORF}^{-}$blue clones and $\mathrm{ORF}^{+}$white clones might initially seem puzzling, but $\mathrm{ORF}^{-}$blue clones were found to contain internal in-frame restart codons, which were in at least some cases preceded by fortuitous

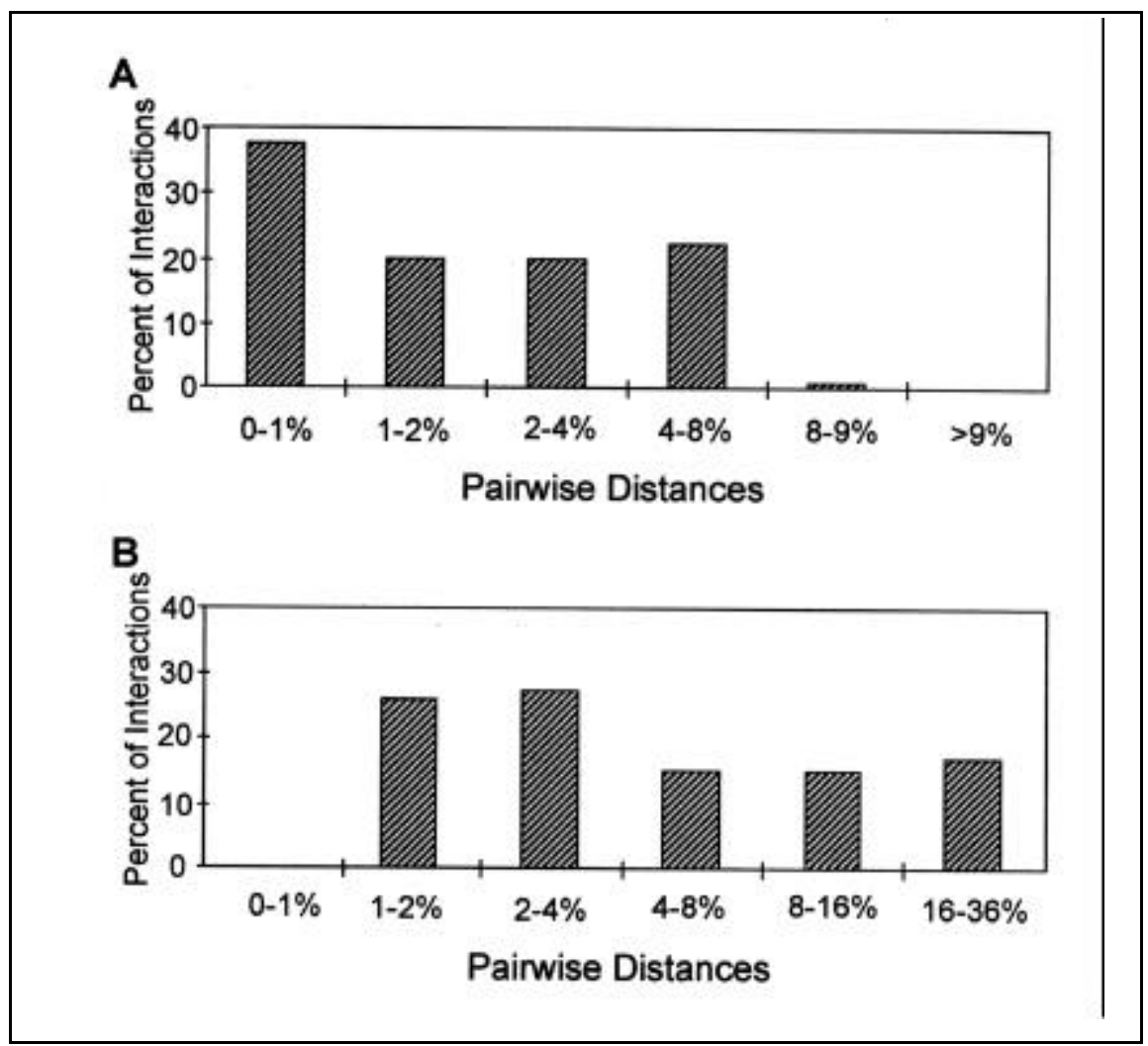

Figure 3. Sequence divergences between L1s. (A) Pairwise distances between L1s from blue clones. (B) Pairwise distances between L1s from white clones. The Y-axis gives the percent of the total pairwise interactions between any two elements that fall within the indicated pairwise distances, or percent sequence differences, shown on the $\mathrm{X}$-axis. 


\section{Research Report}

Shine-Dalgarno signals. $\mathrm{ORF}^{+}$white clones presumably exist because they produce lower levels of functional $\beta$ galactosidase $(\beta$-gal) fusion protein. Reasons for this could include the presence of L1 variants or mutations that reduce the translation elongation rate or fusion proteins that do not fold properly. Upon restreaking, blue colonies reproducibly gave rise to blue colonies and white colonies gave rise to white colonies irrespective of their ORF content.

Do blue colonies indeed contain a more recently inserted (younger) set of L1 sequences than white colonies? Younger L1s should show less sequence divergence between elements than older L1s because they have had less time to accumulate mutations than those copies that were inserted at an earlier time. We therefore looked at pairwise distances (percent sequence difference) between L1s from the predominant L1 lineage in $P$. maniculatus. We only used L1s from Lineage 1, or those which predated Lineage 1, because some of the differences in L1s from different lineages (i.e., produced from different active master elements) would be due to divergence in the masters from which they were derived rather than mutations that accumulated after they inserted into the genome [see Casavant et al. (4) for an explanation of L1 lineages in $P$. maniculatus]. Figure 3 shows the ranges of pairwise distances obtained between L1 sequences from blue clones (3A) and between L1 sequences from white clones (3B). Thirty-seven percent of the pairwise distances between L1s from blue clones show less than $1 \%$ difference in their sequences, while none of the white clones yield L1s with such small pairwise distances. The same trend is seen upon comparison of average pairwise distances for all Lineage 1 L1s. The average pairwise distance between all L1s derived from blue clones is $2.6 \%$, while the average pairwise distance between L1s derived from white clones is $9.2 \%$. This suggests that use of the color screen indeed leads to identification of a substantially younger set of elements. Phylogenetic analysis (not shown) also indicates that elements derived from blue clones tend to be substantially younger than elements derived from white clones.

When we compared the L1s isolated with this technique to previously isolated L1s from $P$. maniculatus, we found that the new technique allows us to identify younger elements and to identify them much more easily, even though some of the previously isolated elements were identified in searches specifically tailored for isolation of young elements (4). How young are the most recently inserted elements? Among the 136 pairwise comparisons between blue clones here, 10 show a pairwise distance of only $0.2 \%$ (a single base pair difference) and two pairs are identical, suggesting that they represent extremely recent insertion events. When analyzing such elements, one should bear in mind some of the basic problems associated

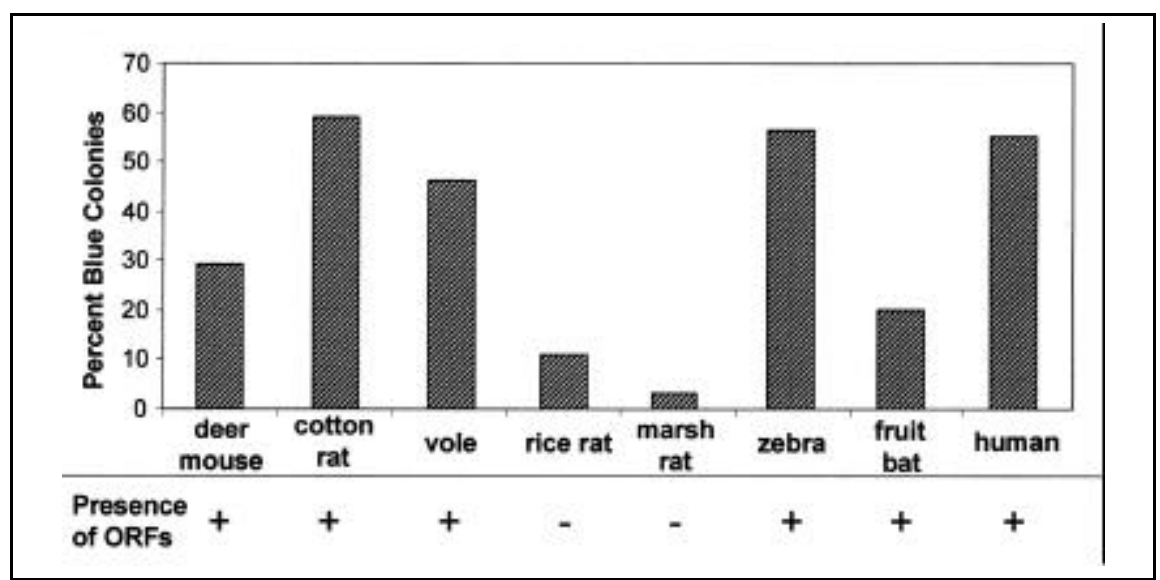

Figure 4. ORF screening allows rapid scanning of L1 activity from a range of mammals. Deer mouse, P. maniculatus; cotton rat, Sigmodon mascotensis; vole, Microtus arvalis; rice rat, Oryzomys palustris; marsh rat, Holochilus brasiliensis; Hartmann's mountain zebra, Equus zebra; Mexican fruit bat, Artibeus jamaicensis; and human, Homo sapiens. with all PCR-based approaches. Clones this similar may in some cases be artifacts produced by PCR errors, while identical clones can arise as multiple amplification products from a single element. There are times when older elements, rather than younger ones, are desired for complete analysis of the evolutionary history of L1s within a species. The availability of such elements from this screen is demonstrated by the extreme sequence differences of up to $36 \%$ between white clones.

We have seen variation in the populations of elements amplified in independent experiments, but a broad spectrum of elements appears to be obtained from every amplification. For example, $P$. maniculatus is known to contain two major L1 lineages, with Lineage 1 being present at a substantially higher copy number in the genome than Lineage 2 (4). Phylogenetic analysis (not shown) indicates that we recovered both of these lineages during independent amplifications. Even though the percentage of clones belonging to each lineage varied between amplifications, Lineage 1 appeared more commonly in both. In addition, elements from one specific amplification do not cluster into particular clades but interdigitate with elements from separate amplifications, as would be expected. Nonetheless, during amplification of mixed populations, one should always bear in mind that the am plified population does not necessarily reflect the genomic population because some sequences will always be preferentially amplified over others because of such factors as concentration of specific primer subsets, amplification conditions that favor certain sequences, the possibility of target divergence from the primer set, and stochastic effects. Younger elements in particular will be preferentially amplified because they have not suffered the mutational divergence from an active element that occurs with time in older elements.

We have found that this technique allows rapid, easy isolation of this region of L1 elements from a broad phylogenetic range of mammals and has the possibility of giving a quick preliminary test for L1 activity in these animals. With every species amplified, we have obtained L1s. Figure 4 shows a selection of some of these species, indicating that 
we see a wide range for the percent blue clones produced. When we have seen the level of blue clones reduced to less than $15 \%$, as with many species of rodents of the closely related genera Oryzomys and Holochilus, we have been unable to find any L1s with ORFs in spite of extensive searches by this and other procedures. See Casavant et al. (3) for further characterization of blue clones from Oryzomys. These observations on the Oryzomys and Holochilus species are part of the data supporting the surprising suggestion that these rodents are the first identified mammals in which L1 activity has ceased (3).

This technique for easy isolation and delineation of a portion of young and old L1 elements using minute amounts of DNA from a wide range of mammals will greatly facilitate the use of these elements as extremely powerful markers to determine the evolutionary history of mammals $(7,21)$. The use of endogenous retroviral sequences for constructing mammalian phylogenies (9) should also be simplified by using this technique with modified primers. Since it allows the easy isolation of elements from such a wide range of mam mals, this method should be extremely useful for studies into the mode and rate of retroelement evolution throughout much of the mammals.

\section{ACKNOWLEDGMENTS}

This work was supported by National Institutes of Health (NIH) grant no. GM38737 to H.A.W. We thank an anonymous reviewer of our last NIH renewal for the suggestion to use a color screening technique for ORFs. DNA was provided by Dr. Robert J. Baker and The Museum, Texas Tech University.

\section{REFERENCES}

1.Ausubel, F.M., R. Brent, R.E. Kingston, D.D. Moore, J.G. Seidman, J.A. Smith and K. Struhl (Eds.). 1989. Current Protocols in Molecular Biology. Green Publishers/WileyInterscience, New York.

2.Benit, L., J.B. Lallemand, J.F. Casella, H. Philippe and T. Heidmann. 1999. ERV-Lelements: a family of endogenous retroviruslike elements active throughout the evolution of mammals. J. Virol. 73:3301-3308.

3.Casavant, N.C., L.A. Scott, M.A. Cantrell, L.E. Wiggins, R.J. Baker and H.A. Wichman. 2000. The end of the LINE? Lack of recent L1 activity in a group of South American rodents. Genetics 154:1809-1817.

4.Casavant, N.C., A.N. Sherman and H.A. Wichman. 1996. Two persistent LINE-1 lineages in Peromyscus have unequal rates of evolution. Genetics 142:1289-1298.

5.DeBerardinis, R.J., J.L. Goodier, E.M. Ostertag and H.H. Kazazian, Jr. 1998. Rapid amplification of a retrotransposon subfamily is evolving the mouse genome. Nat. Genet. 20:288-290.

6.Friedlander, A. and R. Patarca. 1999. Endogenous proviruses. Crit. Rev. Oncog. 10:129159.

7.Furano, A.V. and K. Usdin. 1995. DNA "fossils" and phylogenetic analysis. Using L1 (LINE-1, long interspersed repeated) DNA to determine the evolutionary history of mam mals. J. Biol. Chem. 270:25301-25304.

8.Hutchison III, C.A., S.C. Hardies, D.D. Loeb, W.R. Shehee and M.H. Edgell. 1989. LINEs and related retroposons: long interspersed repeated sequences in the eucaryotic genome, p. 593-617. In D.E. Berg and M.M. Howe (Eds.), Mobile DNA. American Society for Microbiology, Washington, DC.

9.Johnson, W.E. and J.M. Coffin. 1999. Constructing primate phylogenies from ancient retrovirus sequences. Proc. Natl. Acad. Sci. US A96:10254-10260.

10.Kass, D.H., F.G. Berger and W.D. Dawson.
1992. The evolution of coexisting highly divergent LINE-1 subfamilies within the rodent genus Peromyscus. J. Mol. Evol. 35:472-485.

11.Kazazian, Jr., H.H., C. Wong, H. Youssoufian, A.F. Scott, D.G. Phillips and S.E. Antonarakis. 1988. Haemophilia A resulting from de novo insertion of $\mathrm{L} 1$ sequences represents a novel mechanism for mutation in man. Nature 332:164-166.

12.Loeb, D.D., R.W. Padgett, S.C. Hardies, W.R. Shehee, M.B. Commer, M.H. Edgell and C.A. Hutchison III. 1986. The sequence of a large L1Md element reveals tandemly repeated $5^{\prime}$ end and several features found in retrotransposons. Mol. Cell. Biol. 6:168-182.

13.Martin, F.H., M.M. Castro, F. Aboul-ela and I. Tinoco, Jr. 1985. Base pairing involving deoxyinosine: implications for probe design. Nucleic Acids Res. 13:8927-8938.

14.Martin, S.L. 1995. Characterization of a LINE-1 cDNA that originated from RNA present in ribonucleoprotein particles: implications for the structure of an active mouse LINE-1. Gene 153:261-266.

15.McPherson, M.J., B.D. Hames and G.R. Taylor. 1995. PCR 2: A Practical Approach. IRL Press at Oxford University Press, Oxford.

16.Modi, W.S. 1996. Phylogenetic history of LINE-1 among arvicolid rodents. Mol. Biol. Evol. 13:633-641.

17. Rossolini, G.M., S. Cresti, A. Ingianni, P. Cattani, M.L. Riccio and G. Satta. 1994. Use of deoxyinosine-containing primers vs degenerate primers for polymerase chain reaction based on ambiguous sequence information. Mol. Cell. Probes 8:91-98.

18.Sassaman, D.M., B.A. Dombroski, J.V. Moran, M.L. Kimberland, T.P. Naas, R.J. DeBerardinis, A. Gabriel, G.D. Swergold and H.H. Kazazian, Jr. 1997. Many human L1 elements are capable of retrotransposition. Nat. Genet. 16:37-43.

19.Van Den Bussche, R. and H.A. Wichman. 1992. In search of retrotransposons: exploring the potential of the PCR. BioTechniques $13: 258-264$.

20.Vanlerberghe, F., F. Bonhomme, C.A. Hutchison and M.H. Edgell. 1993. A major difference between the divergence patterns within the Lines-1 families in mice and voles. Mol. Biol. Evol. 10:719-731.

21.Verneau, O., F. Catzeflis and A.V. Furano. 1998. Determining and dating recent rodent speciation events by using L1 (LINE-1) retrotransposons. Proc. Natl. Acad. Sci. USA 95:11284-11289.

22.Xiong, Y. and T.H. Eickbush. 1990. Origin and evolution of retroelements based upon their reverse transcriptase sequences. EMBO J. 9:3353-3362.

Received 10 April 2000; accepted 25 July 2000 .

Address correspondence to:

Dr. Holly A. Wichman

Department of Biological Sciences

University of Idaho

Moscow, ID 83844-3051, USA

e-mail: hwichman@uidaho.edu 